\title{
Brain Activity and Movement Cognition - Vibratory Stimulation-Induced Illusions of Movements
}

\author{
Shu Morioka \\ Kio University \\ Japan
}

\section{Introduction}

Feedback information on movement from the musculoskeletal system plays an important role in appropriate control of movement as well as acquisition of new movements. Especially, information on limb movement and location of the movement conveyed from the musculoskeletal system to the brain is assumed to play an essential role in the creation of a body image in the brain. When human limbs actually move, multiple pieces of sensory information are input into the brain by the skin, muscles, joints, etc. Information from muscle spindles has been demonstrated to be most important for cognition of limb movement. ${ }^{1,2}$

A sense of movement can be intentionally aroused by imposing vibratory stimulus to the tendon, regardless of whether actual movement takes place. Such stimulation makes muscle spindles discharge signals as afferent impulses toward the brain. A human recipient of these impulses feels movement of the body in an illusion through perception of muscle extension. (Fig. 1) ${ }^{1)}$ This illusion is elicited mainly by activation of Ia fibers from the muscle spindle. In usual movements, muscle spindles are activated as the muscle is extended. Therefore, if vibratory stimulus activates muscle spindles, information on movement is conveyed to the brain as if the muscle were extended. Hence, a subject can experience limb movement during vibratory stimulation of the tendon despite the absence of actual limb movement. Specifically, tendon stimulation by vibration can induce a sense of movement in the absence of real movement. If brain activity at that moment can be detected, it is possible to study brain activity at the time movement is perceived.

In fact, it was demonstrated that the motor area contralateral to the stimulated limb was activated when illusory hand joint extension was produced by vibratory stimulation of the hand extensor tendon. ${ }^{3}$ It was, however, reported that the brain was predominantly active in the right hemisphere irrespective of which hand was stimulated as far as premotor and parietal areas were concerned.4) Such vibratory stimulation-based illusory movementinduced activity of the brain is nearly identical to that occurring during real movement; this is especially true of premotor and supplementary motor areas, the cerebellum, and the parietal lobe.5) On the other hand, when the hand extensor tendon is stimulated with vibration while an object is being held by the hand, an illusory sense of both hand flexion 


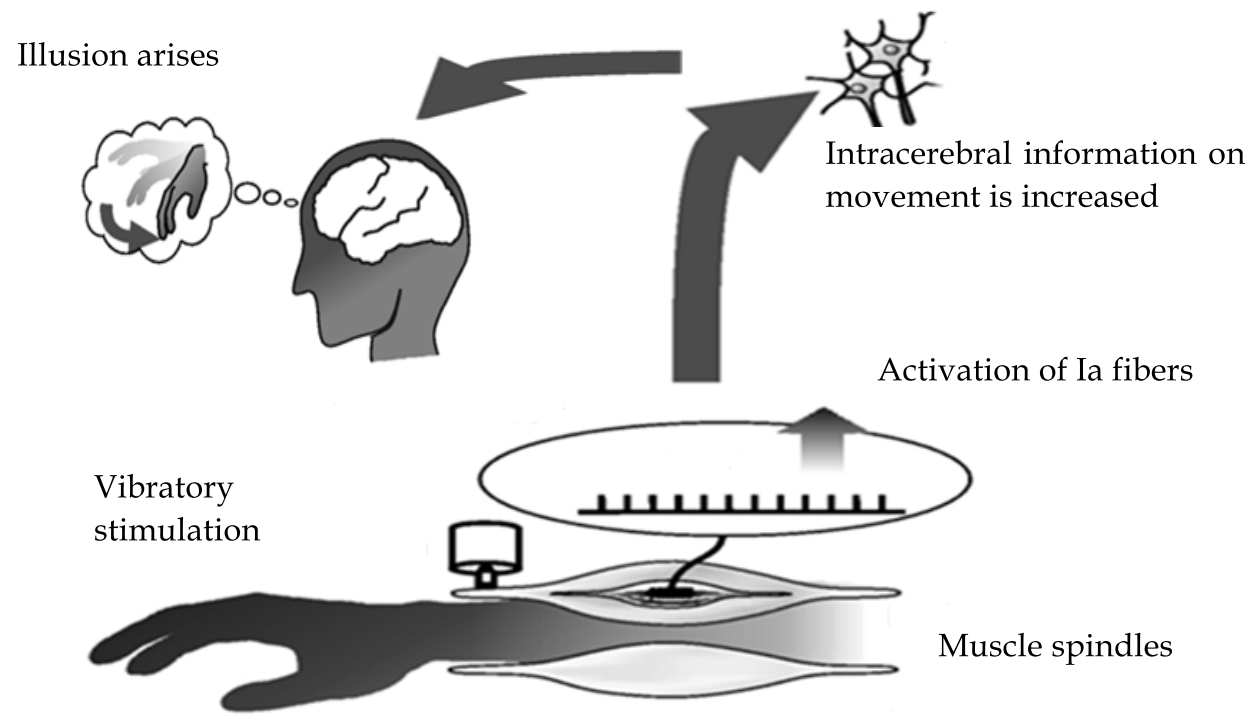

Fig. 1. Schema of tendon stimulation-induced illusory movement

and of the object moving in the same direction is felt. When an object is felt to move in an illusion, the left hemisphere is predominantly activated and regions of interest are the lobule of the inferior parietal lobe and Brodmann's area 44/45.6-8)

However, when an object is placed within the angle of a self-experienced illusion without the object being grasped, it remains to be clarified how much such visual information influences the degree of illusory movement and how the brain is activated. This chapter reports such influences as well as brain activity as explored by functional near-infrared spectroscopy (fNIRS).

\section{Methods}

\subsection{Subjects}

Eight healthy adults in their 20s were initially examined for the intensity of the sensation of illusory movement after vibratory stimulation of the hand extensor tendon using the vibratory stimulation apparatus described below. Intensity was graded on a 5-stage scale. The 7 individuals who were assessed as having scale 4 (strong illusory sensation) or greater were studied. These 7 subjects were observed for brain activity when they experienced illusory sensations and were found to have increased blood flow in the area that responded to illusory movement sensation.6) They were therefore considered eligible for this study. According to the Helsinki Declaration, they were given an explanation about the purpose of this study before providing written informed consent.

\subsection{Vibratory stimulation apparatus}

We used the SL-0105 LP (Asahifactory Corp., Tokyo) (Fig. 2) for vibratory stimulation under conditions of frequency of $80 \mathrm{~Hz}$ and intensity of $0.2-0.3$, which was reported to most 


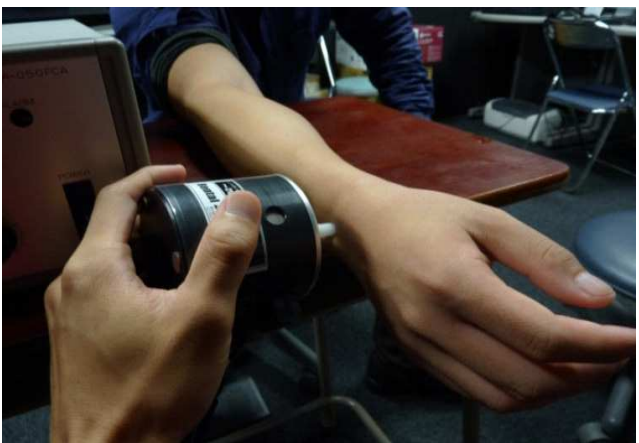

a)

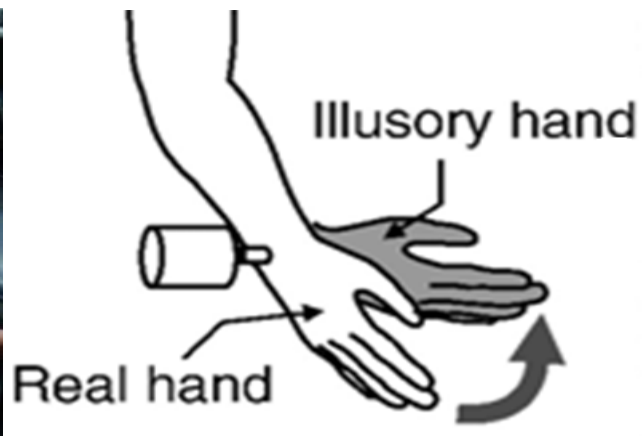

b)

a) Apparatus is in contact with the hand extensor tendon to elicit an illusion of hand joint flexion. (Situation 1)

b) Illustration of illusory movement.

Fig. 2. Application of vibratory stimulus

efficiently excite illusions by an earlier study.4) There were two experimental situations. In situation 1, vibratory stimulation was imposed to the hand extensor tendon without placing an object near the subject; In situation 2, stimulation was similarly imposed with an object (umbrella) standing upright within range of illusory flexion. (Fig. 3) In either situation, the skin near the tendon was stimulated, which served as a control. The purpose of this stimulation was to acquire data on brain activity that was elicited when skin irritation receptors (Meissner corpuscles, Pacinian corpuscles, etc.) were excited that could be subtracted from data acquired by tendon stimulation so that pure illusory brain activity could be calculated.

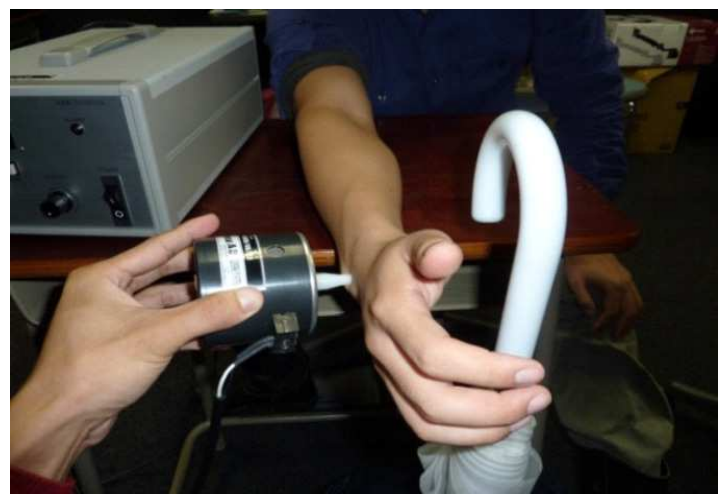

Fig. 3. An object (umbrella) is placed within range of illusory movement. Situa (tion 2)

\subsection{Protocol of tasks}

A task to create an illusion for a period of more than 20 seconds was imposed between two 10 -second resting times. This session was uninterruptedly repeated under each situation. The illusory flexion angle was estimated as follows. After accomplishment of a task, a 
subject was requested to reenact with an actual motion the illusory limb movement and the flexion angle was measured with an electric geniometer (SG150, Biometrics, Phoenix).

\subsection{Measurement by fNIRS}

Cerebral blood flow was measured using fNIRS FOIRE-3000 (Shimazu Co., Kyoto). (Fig. 4) Probes were fixed using a flexible holder to cover the entire head, which was placed on the surface of the scalp covering the bilateral frontal and parietal lobes. (Fig. 4) The distance between the pair of emission and detector probes was $3.0 \mathrm{~cm}$ and the pair was set as a channel (ch). The Cz of the International 10-20 method for EEG was set at the point where a line drawn between the bilateral external auditory pores and a perpendicular line from the nose intersect. Emission probe \#7 was placed at the $\mathrm{Cz}$ to allow measurement sites be shared equally by all the subjects. Fifteen emission probes (E) and detection probes (D), respectively, were placed in a 5X6 arrangement in order starting from the left forehead. It total, 49 channels were provided. (Fig. 5) To prevent the hair from protruding, fibers (multicomponent glass band fibers) were set through the hair to a fiber holder to ensure that their tips contacted the scalp.

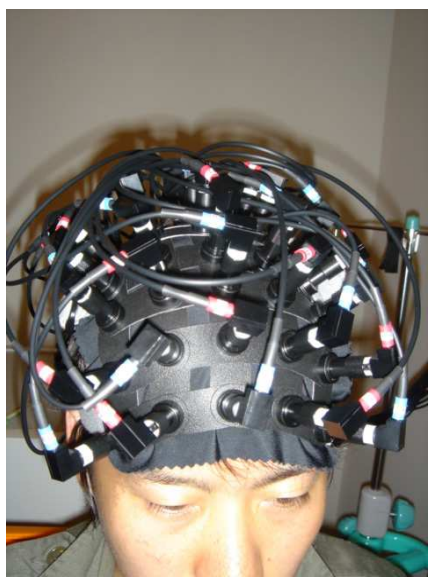

Emission and detector probes are placed at a distance of $3 \mathrm{~cm}$ from each other and each pair of probes works as a channel.

Fig. 4. Subject wearing flexible probe holder covering the entire scalp.

\subsection{Data analysis}

Brain active sites were identified using 3 SPACE FASTRAK (POLHEMU Co., Vermont) and were superimposed on images obtained by magnetic resonance imaging (MRI) using FUSION imaging software (Shimazu, Co., Kyoto).

Oxidized hemoglobin (oxy-Hb) levels at tendon stimulation acquired from 3 continuous task sessions were averaged to get an arithmetic mean from which another arithmetic mean, that which was similarly acquired as the skin was stimulated, was subtracted. Final values were statistically analyzed. Since it is known that there is a time lag of several seconds between the onset of neural activity and change in cerebral blood flow as detected by fNIRS, 


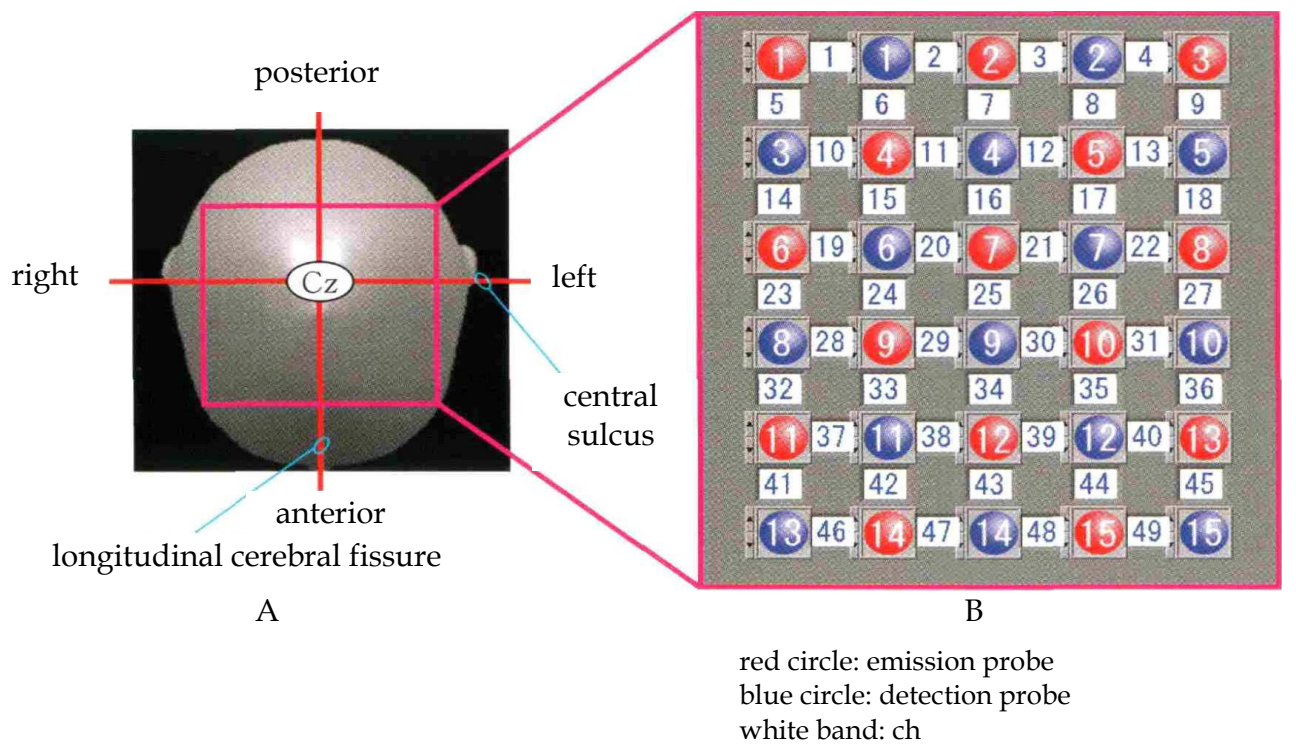

Emission probe \#7 was placed at the $\mathrm{Cz}$ (panel A) as defined by the International 10-20 method so that measurement sites were the same in all subjects (panel B).

Fig. 5. Schematic arrangement of emission and detection probes.

levels of oxy-Hb acquired over the first 5 seconds were discarded and levels obtained over another 5 seconds were subjected to analysis during both tasks and resting times. Furthermore, the issue of optical path length was solved by calculation of effect sizes (=[(an arithmetic mean value of oxy-Hb levels at a task ) - (that at a resting time)]/standard deviation at a resting time).9) In addition, effect sizes were separately averaged for each region of interest. Specifically they were analyzed as follows: ch 1, 2, 5, 6, 10, and 11 representing the right parietal lobe; ch 3, 4, 8, 9, 12, and 13, the left parietal lobe; ch $14-27$, bilateral sensorimotor areas; ch 28, 29,32, 33, 37, 38, 41, 46, and 47, the right premotor area; and ch 30, 31, 35, 36, 44, 45, 48, and 49, the left premotor area. (Fig. 6)

Differences in brain activity between situations 1 and 2 were tested with Wilcoxon matchedpairs signed-ranks test. Angles of illusions were analyzed by the t-test. Pearson's correlation coefficients were calculated to examine relations between differences in the angles of illusions between situations 1 and 2 and similar differences in the brain activity. Level of significance was set as $\mathrm{p}<0.05$.

\section{Results}

Angles of the illusion were significantly decreased in situation 2 compared to situation 1 (Fig. 7, p < 0.05), while brain activity in the premotor area as assessed by effect size was reduced in situation 2. To confirm this finding, cerebral areas related to these channels were superimposed on MRI images and were identified as the right premotor area. (Fig. 8) On the other hand, there was a positive relationship, although not significant $(\mathrm{p}=0.17)$, between a decrease in illusion angles and a reduction in activity in the right premotor area $(\mathrm{r}=0.58)$. 


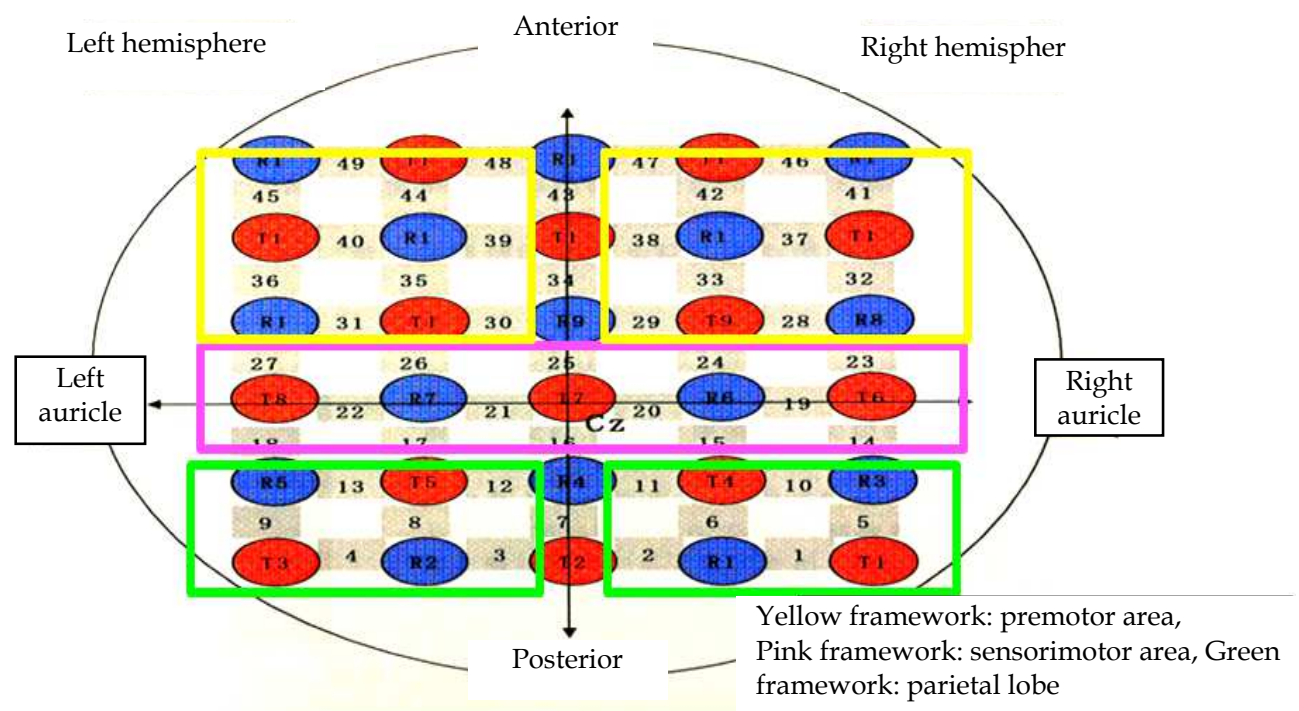

Channels were grouped into 5 regions and effect sizes from each region were averaged.

Fig. 6. Schema of regions of interest analysis

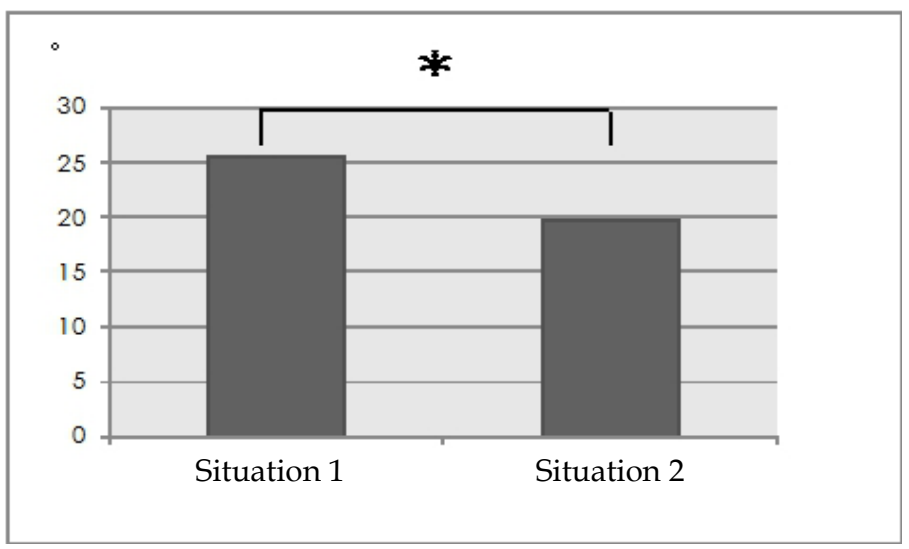

* $\mathrm{P}<0.05$

Situation 1: No object is presented, Situation 2: An object is presented.

There is a significant reduction of flexion angles in situation 2 compared to situation 1 . In other words, object presentation reduces the degree of illusory hand joint flexion.

Fig. 7. Differences in angles of hand joint flexion between Situations 1 and 2 


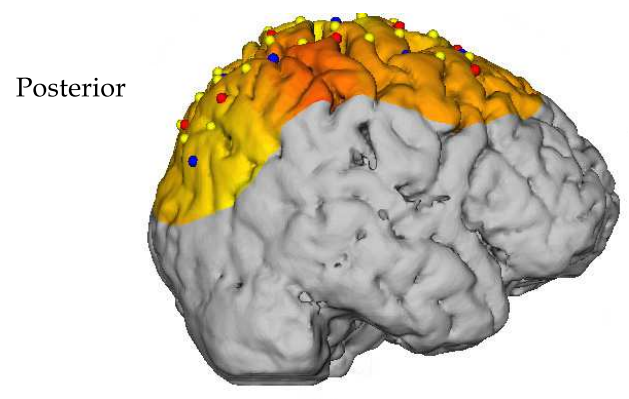

Situation 1

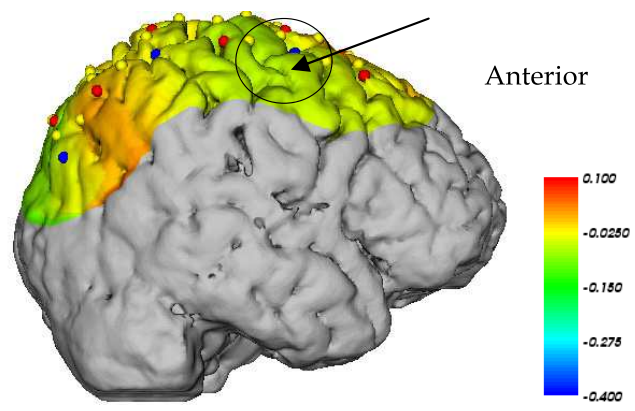

Situation 2

Warm colors denote a relative increase in blood flow, while cold colors denote a relative decrease in blood flow.

In situation 2 , in the right premotor area there is a reduction in activity compared to situation 1 . (Arrow within circle)

Fig. 8. Comparison of cerebral cortical activities between situations 1 and 2 .

\section{Discussion}

The present results clarified that the angle of illusion was decreased by placing a visible object within the range of illusory movement of the subject's own hand. It is therefore evident that visual sensation can modify illusory movement, indicating that the environment largely influences movement cognition. The effect size of the channels corresponding to the right premotor area were significantly reduced when vibratory stimulation was given in the presence of a visible object in comparison with absence of an object. In summary, the reduction in activity of the right premotor area was assumed to have diminished illusory movement as suggested by a positive correlation between intensities of the area activity and those of the illusion. That this correlation was not significant may have been because of the small subject population. It was thus clear that the activity of the premotor area of the right hemisphere influenced perception of movement and that the degree of activation of the right premotor area was involved in formation or suppression of movement perception.

The right premotor area is the region responsible for bodily self-attribution as evidenced by Ehrsson et al.10) Evidence of the predominance of the right hemisphere in illusory perception of limb movement elicited by tendon vibration was also reported.8) Our present study added new information that a decline in activity in the premotor area suppressed illusory movements. Pinocchio's illusion ${ }^{11)}$ helps explain this observation. When an illusory movement of elbow extension is provoked by vibratory stimulation with your nose being pinched, you feel as if your nose got longer. This phenomenon occurs to clear up a contradiction between illusory limb movements and constant input of tactile sensation from the nose. In this study, the subject should have touched an object due to illusory movements if it had existed within the illusory angle of the subject's own hand. Our results suggested that the degree of illusory movements was lowered due to lack of tactile sensation and that the right premotor area might be involved in variability of illusory movements.

From these results it was clarified that movement perception based on activity of muscle spindles was easily modified by a change in visual information even though the intensity of 
vibratory stimulus was fixed. Namely, bottom-up processing of sensory information from afferent fibers is influenced by its top-down processing. On the other hand, it remains unclear what directly reduced the activity of the right premotor area. To answer this question, it is necessary to examine if an object presented outside the angle of illusion changes the degree of illusory movements. In addition, it will be necessary to study the status of activities of visual cortical areas as well as of prefrontal areas rather than study premotor areas.

\section{References}

[1] Roll JP, et al: Kinaesthetic role of muscle afferents in man, studied by tendon vibration and microneurography. Exp Brain Res 47: 177-190, 1982

[2] Roll JP, et al: Alteration of proprioceptive messages induced by tendon vibration in man: a microneurographic study. Exp Brain Res 76: 213-222, 1989

[3] Naito E et al: Illusory arm movements activate cortical motor areas: a positron emission tomography study. J Neurosci 19(14):6134-6144, 1999

[4] Naito E et al: Kinesthetic illusion of wrist movement activates motor-related areas. Neuroreport 12: 3805-3809, 2011

[5] Naito E, et al: Internally simulated movement sensations during motor imagery activate cortical motor areas and the cerebellum. J Neurosci 22: 3683-3691, 2002

[6] Naito E, et al: Somatic sensation of hand-object interactive movement is associated with activity in the left inferior parietal cortex. J Neurosci 26: 3783-3790, 2006

[7] Naito E, et al: Human superior parietal lobule is involved in somatic perception of bimanual interaction with an external object. J Neurophysiol 99: 695-703,2008

[8] Naito E, et al: Dominance of the right hemisphere and role of area 2 in human kinesthesia.J Neurophysiol 93 :1020-1034,2005

[9] Schroeter L, et al : Age dependency of the hemodynamic response as measured by functional near-infrared spectroscopy.Neuroimage19: 555-564,2003

[10] Ehrsson HH, et al: That's my hand! Activity in premotor cortex reflects feeling of ownership of a limb. Science 305: 875-877,2004

[11] Lankner JR: Some proprioceptive influences on the perceptual representation of body shape and orientation. Brain 111:281-297,1988 


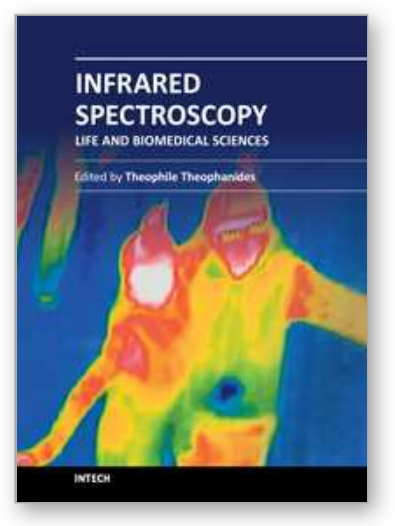

\author{
Infrared Spectroscopy - Life and Biomedical Sciences \\ Edited by Prof. Theophanides Theophile
}

ISBN 978-953-51-0538-1

Hard cover, 368 pages

Publisher InTech

Published online 25, April, 2012

Published in print edition April, 2012

This informative and state-of-the art book on Infrared Spectroscopy in Life sciences designed for researchers, academics as well as for those working in industry, agriculture and in pharmaceutical companies features 20 chapters of applications of MIRS and NIRS in brain activity and clinical research. It shows excellent FT-IR spectra of breast tissues, atheromatic plaques, human bones and projects assessment of haemodynamic activation in the cerebral cortex, brain oxygenation studies and many interesting insights from a medical perspective.

\title{
How to reference
}

In order to correctly reference this scholarly work, feel free to copy and paste the following:

Shu Morioka (2012). Brain Activity and Movement Cognition - Vibratory Stimulation-Induced Illusions of Movements, Infrared Spectroscopy - Life and Biomedical Sciences, Prof. Theophanides Theophile (Ed.), ISBN: 978-953-51-0538-1, InTech, Available from: http://www.intechopen.com/books/infrared-spectroscopy-life-andbiomedical-sciences/human-brain-activity-and-motor-perception

\section{INTECH}

open science | open minds

\section{InTech Europe}

University Campus STeP Ri

Slavka Krautzeka 83/A

51000 Rijeka, Croatia

Phone: +385 (51) 770447

Fax: +385 (51) 686166

www.intechopen.com

\section{InTech China}

Unit 405, Office Block, Hotel Equatorial Shanghai

No.65, Yan An Road (West), Shanghai, 200040, China

中国上海市延安西路65号上海国际贵都大饭店办公楼 405 单元

Phone: +86-21-62489820

Fax: +86-21-62489821 
(C) 2012 The Author(s). Licensee IntechOpen. This is an open access article distributed under the terms of the Creative Commons Attribution 3.0 License, which permits unrestricted use, distribution, and reproduction in any medium, provided the original work is properly cited. 